\title{
诱发电位的潜伏期校准迭加平均 处理方法研究
}

\author{
万柏 坤 \\ (天津理工学院物理系, 天津 300191)
}

\section{关镜词诱发电位、潜伏期、迭加平均}

迭加平均（average）是提取淹没在噪声本底中周期性重复出现信号的有效方法. 以刺 激时刻为基准的时域迭加平均（time-locked average, TLA) 已成为从自发脑波（EEG）背 景中提取各种诱发电位 (evoked potential) 的传统方法. 其前提是假定各反应波峰的潜伏 期 (latency) 相对刺激时刻基本固定. 但大量实验结果和临床实践表明, 诱发电位中的潜伏 期在每次刺激后并非严格不变. 故上述 TLA 方法并不能十分有效地获得诱发电位信号. 因 此, 预先检测诱发电位的潜伏期, 并对其漂移作修正, 然后再作迭加平均, 当可获得更为有效的 处理结果 ${ }^{[1-2]}$. 作者与武者利光教授提出了用相位滤波器 (phase-only filter) 检测诱发电位 潜伏期, 进而提出诱发电位的潜伏期校准迭加平均（latency-aligned average, LAA) 处理新 方法 ${ }^{[3]}$.

相位滤波器是仅保留原信号傅里叶变换中的相位信息，而将其振幅信息白噪声化的非线 性变换。鉴于相位信息在大脑诱发电位中的重要作用,且计算机模拟结果表明,相位滤波器能 增强诱发电位信号中的反应波峰而不改变原波峰的潜伏期.故可用该方法检测潜伏期,经校准
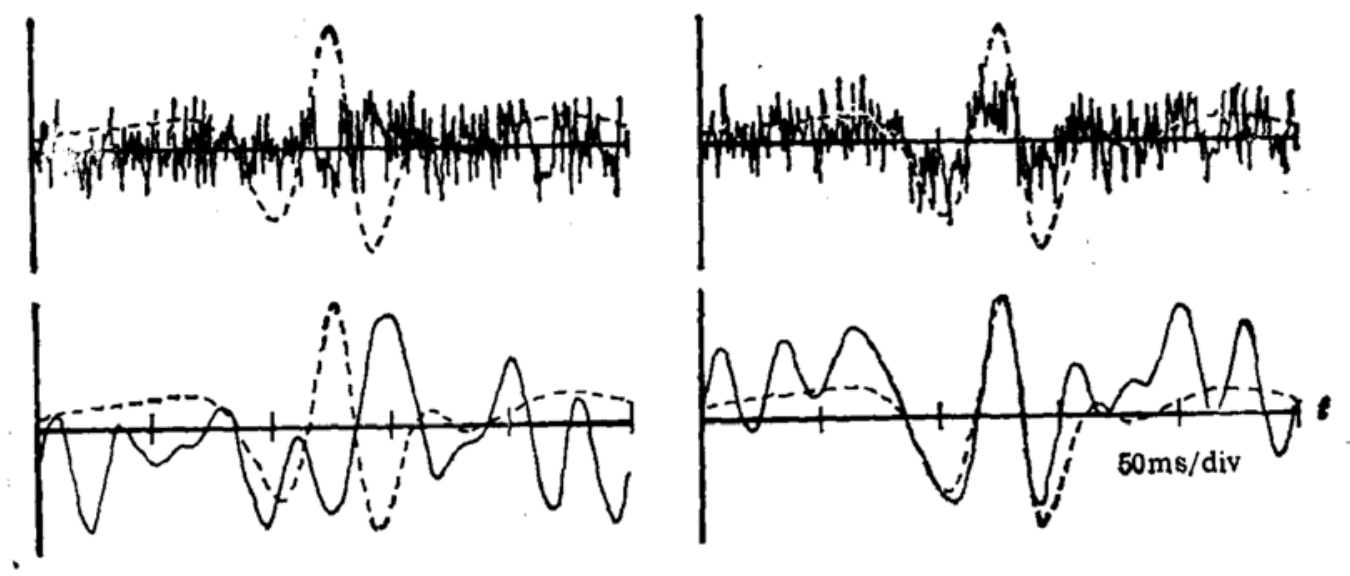

图 1 相位滤波效果示例

图中上部为被检测信号, 下部为相位波誩号; 左边为白噪声情况，右边为 白噪声与诱发电位 (VEP) 波形合成信号; VEP 波形及其相位滤波信号如 虚线所示. 为显示方便, 绘图时将㖼声揊度适当减小

本文 1990 年 4 月 17 日收到.

第 16 期

科学通报 


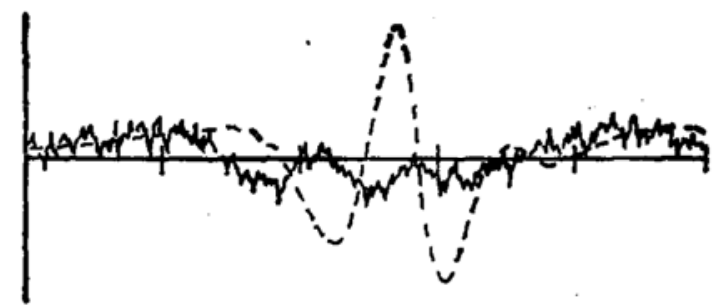

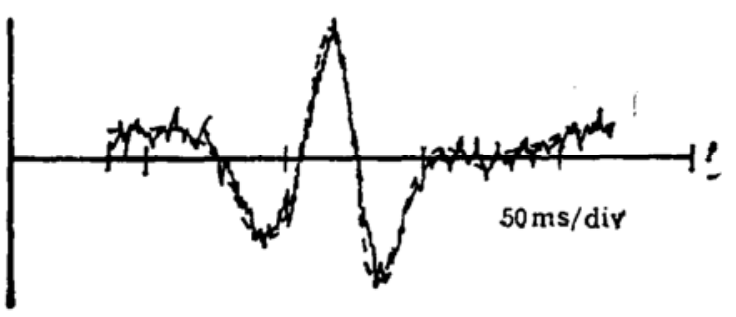

图 2 TLA 和 LAA 处理效果比较之一 将白噪声与在时轴上随机漂移的诱发电位 (VEP) 波 形所合成信号分别作 TLA 和 LAA 处理. 图中上 部为 TLA 结果, 下部为 LAA 结果; 虚线为 VEP 波形，迭加 40 次; 下边图形两端空缺是为避开潜伏期 最大偏移所致

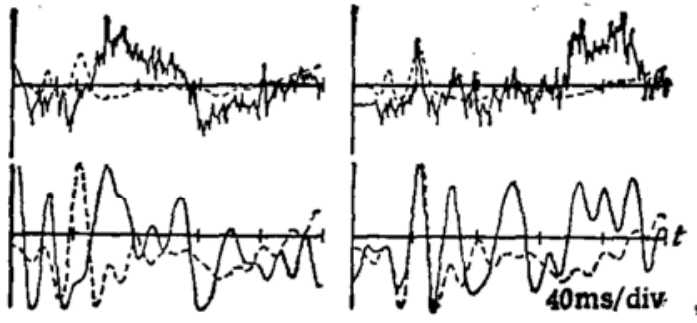

图 3 相位滤波检测示例

图中上部为被柆测信号, 下部为相位滤波信 昂; 左边为仅有自发脑波 (EEG) 情况,右边 为 EEG 与诱发电位 (SEP) 波形合成信 号;SEP 波形及其相位港波信号如虚线所示

后再作迭加平均, 可望获得比传统的 TLA 方法更有效的结果. 本文仅以计算机模拟为例研

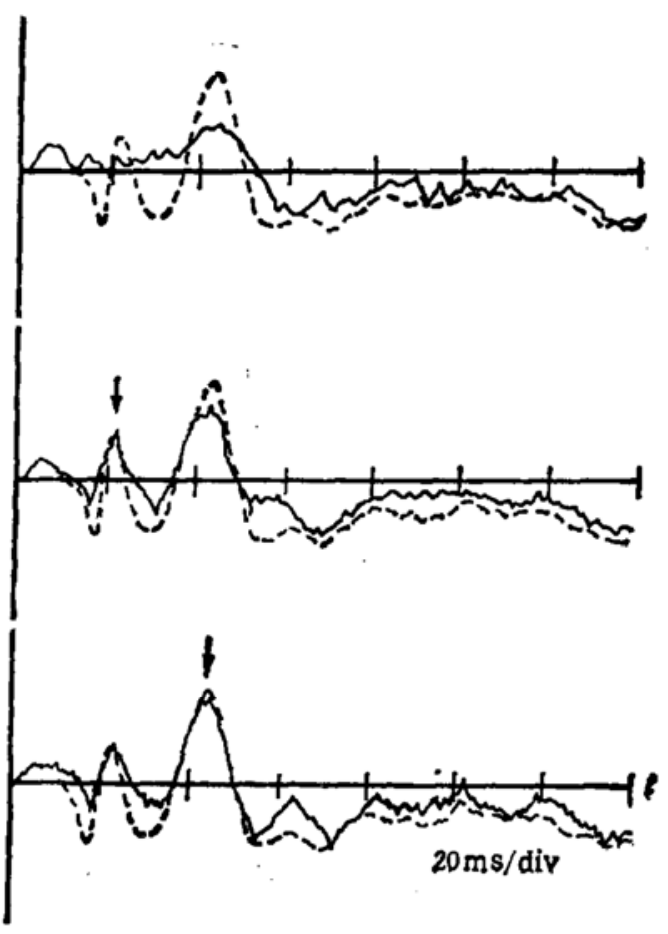

图 4 TLA 和 LAA 处理效果比较之二 将自发脑波 (EEG) 与在时轴上随机漂移的诱发电位 (SEP) 波形所合成信号分别作 TLA 和 LAA 处理. 图中上部为 TLA 结果, 中间及下部为 LAA 结果; 箭头示意对该波峰的潜伏期校准. 应线为 SEP 波形, 迭加 80 次
究 LAA 方法的处理效果.

其一是, 用诱发电位的谐振模型生成诱 发电位波形 (VEP $)^{[4]}$ 再与计算机产生的随: 机白噪声合成为待检测信号。

诱发电位（VEP）信号可由文献 [4] 选: 取适当参数值得到, 波形见图 1 所示。

噪声本底是通过计算机随机数函数产 生, 平均值为零的白噪声. 信噪比约 -12 dB. 采用在谐振正弦波的相移时间参数中 引人随机变量的方法, 可使每次合成待测信; 号中的诱发电位波形在时间轴上作随机漂移 以模拟实际诱发电位潜伏期随刺激而发生的 变化。

图 1 举例说明用相位滤波器检测潜伏期 的结果. 图中左边是仅有白嗓声, 未含诱发 电位信号的情况, 其相位滤波信号亦不出现 与诱发电位波形相对应的波峰, 右边则是白 噪声与诱发电位波形的合成信号. 可以看 出, 其相位滤波信号有一与原信号最大波峰 相对应的峰值.

图 2 是 TLA 和 LAA 处理效果的比. 较. 令诱发电位原信号在时轴上作随机漂移, 分别用 TLA 和 LAA 方法作迭加平均. 上部; 
是 TLA 处理结果, 随着迭加的进行, 原信号波形反而消失殆尽. 而下部用 LAA 方法则仍 可获得较理想的原信号波形. LAA 处理得到的诱发电位波形在时间轴上的位. 置由所迭加的 各次波形中经相位滤波检测到的潜伏期平均值所决定.

其二是, 以实际记录的脑波 (EEG) 作噪声本底, 与多次迭加平均得到的体性感觉诱发电 位 (SEP) 波形合成得到待检测信号. 图 3 示例了用相位滤波器检测潜伏期的结果. 图中左 边仅有 EEG 噪声, 未含 SEP 信号; 右边是 EEG 与 SEP 合成信号, 信噪比约为一 $6 \mathrm{~dB}$. 从 各自的相位滤波信号来看, EEG 背景上迭加 SEP 信号的组合波形, 经相位滤波后在原信号 波峰所对应时刻显现出相应的信号波峰. 而不含 SEP 信息的 EEG 背景所得相位滤波信号 中则无相应的信号峰值出现.

图 4 是用 TLA 和 LAA 处理结果比较. 同样, 由于诱发电位潜伏期的随机漂移, 使 TLA 无法获得原始唀发电位波形; 而 LAA 方法则能及时校准潜伏期, 从而有效地获得真实 信号. 由图可见,无论是以哪个波峰为基准,均能得到较好的处理结果.

综.上所述,用潜伏期校准迭加平均方法 (LAA) 能在潜伏期随机漂移情况下, 从 EEG 噪 声背景中有效地拾取诱发电位信号, 可望为大脑诱发电位信号处理提供新途径.

致谢：本研究得到日本东京工业大学武者利光教授的有益指导和帮助, 谨此致谢。

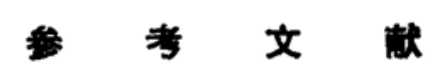

[1] Woody, C. J., Med. Biol. Eng., 1967, 5:539-533.

(2) McGillem, C. D., Aunon. J. I. and Pomalaza, C. A., IEEE Trans. Biomed. Eng., BME-32(1985),371379.

\{3] 万柏坤、武者利光, 信学技报, 86(1986), 272: CAS 086-144.

(4] 唐涂、吕维驺,信号处理, 3(1987)，4: 246-251. 